\title{
Prehospital Adenosine Diphosphate Receptor Blocker Use, Culprit Artery Flow, and Mortality in STEMI: The MADDEC Study
}

\author{
Markus Hautamäki ${ }^{1,2}\left(\right.$ D $\cdot$ Leo-Pekka Lyytikäinen ${ }^{1,2,4} \cdot$ Markku Eskola $^{1,2} \cdot$ Terho Lehtimäki $^{1,3,4} \cdot$ Kjell Nikus $^{1,2,4}$. \\ Niku Oksala ${ }^{1,4,5}$. Juho Tynkkynen ${ }^{6}$. Jussi Hernesniemi ${ }^{1,2,4}$
}

Accepted: 16 May 2021 / Published online: 8 June 2021

(c) The Author(s) 2021

\begin{abstract}
Background and Objective The newer adenosine diphosphate (ADP) receptor blockers ticagrelor and prasugrel are superior to clopidogrel in the long-term management of acute coronary syndrome (ACS). We evaluated the acute performance (prehospital loading) of these ADP receptor blockers in a primary percutaneous coronary intervention (PCI) for an ST-elevation myocardial infarction (STEMI).

Methods In a retrospective, single-center registry study, data on all STEMI patients admitted for their first primary PCI between January 2007 and April 2020 were analyzed $(n=3218)$. The three ADP receptor blockers were mainly used during consecutive periods (clopidogrel 2007-2010, prasugrel 2011-2014, and ticagrelor 2014-2020), and were compared with risk factor-adjusted multivariate logistic regression for acute 3- and 7-day mortality and culprit artery flow before and after PCI. Results Of the 3218 total patients, $47.6 \%(n=1532)$ were treated with ticagrelor, $22.1 \%(n=711)$ were treated with prasugrel, and $30.3 \%(n=975)$ were treated with clopidogrel. The use of ticagrelor or prasugrel as opposed to clopidogrel was associated with better culprit artery flow before PCI (odds ratio [OR] 1.21 for moderate or good flow, $95 \%$ confidence interval [CI] 1.03-1.42, $p=0.022$ ), as well as lower acute mortality (OR 0.66 for 3-day mortality, $95 \%$ CI $0.46-0.95, p=0.025$; and OR 0.71 for 7 -day mortality, $95 \%$ CI $0.52-0.98, p=0.039$ ). The results in regard to acute mortality were highlighted among patients with short treatment delays (disappearing with longer treatment delays; $p<0.05$ for interaction).

Conclusions The newer ADP receptor blockers are associated with lower mortality and better culprit artery flow at presentation when compared with clopidogrel. There are no significant differences between the two newer drugs. As the drugs were mainly used during three consecutive periods, unmeasured confounding related to the development of cardiac care and changes in the population may contribute to the results.
\end{abstract}

Markus Hautamäki

markus.hautamaki@tuni.fi

1 Faculty of Medicine and Health Technology, University of Tampere, Arvo Building, Arvo Ylpön Katu 34, 33520 Tampere, Finland

2 Heart Hospital, Tampere University Hospital, Tampere, Finland

3 Department of Clinical Chemistry, Fimlab Laboratories, Tampere University Hospital, Tampere, Finland

4 Finnish Cardiovascular Research Center Tampere, Tampere, Finland

5 Centre for Vascular Surgery, Tampere University Hospital, Tampere, Finland

6 Department of Radiology, Tampere University Hospital, Tampere, Finland

\section{Key Points}

There are limited data regarding the association between antiplatelet drugs and acute mortality in STEMI patients treated with primary PCI.

This was a retrospective register study of 3218 STEMI patients treated between 1 January 2007 and 15 April 2020.

Newer ADP receptor blockers are associated with lower mortality and better culprit artery flow at presentation when compared with clopidogrel. 


\section{Introduction}

Adenosine diphosphate (ADP) receptor blockers (P2Y 12 inhibitors) are used before an invasive procedure, together with acetylsalicylic acid, to prevent acute thrombosis and subsequent hemostasis (stent thrombosis) during a percutaneous coronary intervention (PCI) and stent implantation [1-4]. This dual antiplatelet therapy (DAPT) has become the therapeutic standard before and after the intervention in the case of an ST-elevation myocardial infarction (STEMI). The timing and dosage depend on the drug used and the certainty of the diagnosis [5-7]. In STEMI, treatment is initiated as early as possible, however in uncertain cases it may be preferable to delay the initiation of P2Y inhibitor therapy while awaiting confirmation of the diagnosis [8]. The results of the randomized and controlled PLATO and TRITON TIMI 38 trials showed that both ticagrelor and prasugrel are superior to clopidogrel in acute coronary syndrome (ACS) in decreasing the risk of cardiovascular death, a recurrent myocardial infarction (MI), or stroke [9-11]. The subgroup analyses of both PLATO and TRITON TIMI 38 showed no significant heterogeneity in the treatment effect between patients with different types of ACS, meaning that the results seemed similar among STEMI patients when compared with nonST-elevation MI patients and patients with unstable angina pectoris $[12,13]$. After these trials, two RCTs have produced head-to-head comparisons of the use of the newer ADP receptor blockers in ACS $[14,15]$. The open-label ISAR-REACT study came to the conclusion that prasugrel was associated with a lower incidence of death, MI, and stroke; however, the study did not clearly compare the drugs head-to-head but rather considered them as different strategies with different antiplatelet medication [14]. In comparison, the results of the randomized and controlled PRAGUE study suggested that there is no evidence of prasugrel being superior to ticagrelor or vice versa [15].

In addition, two meta-analyses were recently published regarding ADP receptor blocker use with ACS and STEMI patients, reporting no significant differences between the drugs as regards either mortality or major adverse cardiac events $[16,17]$. There are no clinical trials dedicated to comparing ticagrelor and prasugrel in STEMI patients, but the results of a large retrospective registry with a propensity matching analysis of over 90,000 patients showed that the use of prasugrel is associated with lower morality when compared with the use of ticagrelor at 30 days and 1 year after a STEMI [18].

Despite the apparent efficacy of prasugrel, its use is somewhat limited by the more stringent limitations; it is contraindicated in patients with a history of transient ischemic attack or stroke and is not generally recommended for the elderly ( $>75$ years) or individuals with a body weight of $<60 \mathrm{~kg}$ [8]. Given the paucity of available data on STEMI patients, and possibly because of the more stringent contraindications of prasugrel, both of these ADP receptor blockers are considered options to be used as primary therapeutic agents in STEMI.

To address these issues, we performed a retrospective registry study to evaluate the use of ADP receptor blockers and their association with culprit artery flow and acute mortality among patients arriving to be treated by primary PCI for STEMI.

\section{Patients and Methods}

This study was conducted as part of the larger retrospective MADDEC (Mass Data in Detection and Prevention of Serious Adverse Events in Cardiovascular Disease) registry study. The MADDEC study combines data from multiple electronic sources on all patients treated at Tays Heart Hospital, Tampere, Finland [19], which is the sole provider of tertiary cardiac care for approximately 0.5 million inhabitants in the region of Pirkanmaa, Finland. In MADDEC, conventional electronic health record (EHR) data are combined with clinical cardiovascular phenotype data collected by treating physicians into a dedicated KARDIO registry. The register contains high-quality phenotype data for risk prediction, with added information over generic hospital EHRs, such as detailed information on patients' hospitalizations, invasive and non-invasive procedures, as well as outpatient visits [20-22].

The present study focuses on patients admitted to the hospital to undergo primary PCI for STEMI between 1 January 2007 and 15 April 2020. During this period, there were 4026 hospitalizations due to STEMI. The analyses were restricted to the first primary PCI episode of each patient within this time frame. Patients initially treated by fibrinolysis $(n=585)$, as well as those who did not receive any oral ADP blocker $(n=223)$, were excluded. During the study period (January 2007-April 2020), the three ADP blockers were routinely used in primary PCI during consecutive periods as the emergency response protocol, and the primary PCI network in STEMI was coordinated centrally (from the study center). The STEMI protocol outlined that, in addition to ADP blockers, all patients were treated with aspirin (oral loading dose of $250 \mathrm{mg}$ ) and intravenous low-molecularweight heparin (enoksaparin) administered at the time of the diagnosis.

\subsection{Data Collection}

For the analyses, the patient data were retrieved from the KARDIO registry and merged with the hospital's EHR 
data. KARDIO data have been collected since 2004, and the data collection is still ongoing. All data in the KARDIO registry have been entered into the system by treating physicians. The data include information about the use of ADP receptor blockers in STEMI and the Thrombolysis in Myocardial Infarction (TIMI) classification of coronary artery flow before and after PCI, based on the angiography imaging [19-22]. For the purposes of this study, missing or incomplete information on the ADP receptor blockers used and coronary artery flow was complemented by a review of the patients' EHRs (including all text records) and angiography images.

\subsection{Main Exposure and Outcome Variables}

The main exposure variable was the information on the ADP receptor blocker agents used. All ADP receptor blockers were administered immediately after the treatment decision on site. With the majority of patients with suspected STEMI in our hospital district, the emergency personnel contact the invasive cardiologist on duty to conduct an emergent teleconsultation for immediate decisions regarding reperfusion therapy and initial medication.

Comparisons were made between patients treated with the newer ADP receptor blockers ticagrelor or prasugrel and the older agent clopidogrel. The main endpoints of the study were acute all-cause mortality (analyzed after 3 and 7 days from the procedure) and the presence or absence of moderate or good culprit artery flow (TIMI 2-3) before or after the primary PCI. Mortality data were provided by the Causes of Death Register maintained by Statistics Finland [23, 24]. Mortality analyses were performed by using two separate time points to confirm the association despite possible changes in ADP receptor blocker treatment during the hospital stay. The mortality data were analyzed for all patients, but the analyses of culprit artery flow were limited only to patients in whom PCI was attempted. The exclusion of patients undergoing $\mathrm{CABG}$ and those treated conservatively from these analyses was done in order to focus the analyses on patients with a clear culprit artery that was considered operable by PCI. This also entailed the exclusion of patients with no other confounding factors due to a complex coronary anatomy (patients treated by $\mathrm{CABG}$ ), patients with a poor overall clinical state upon arrival (palliative treatment strategy), or those with no clearly identifiable culprit artery at angiography (possible other diagnosis, such as a passing cardioembolic STEMI).

\subsection{Statistical Analysis}

The significance of the differences between the subpopulations were tested using the Kruskal-Wallis test (scale variables) and one-way analysis of variance (ANOVA; nominal variables). Multivariate logistic regression analysis was used to retrospectively analyze the association between the use of the initial ADP receptor blocker and the outcome variables. Analyses were adjusted with the following confounding factors: age, sex, presence of diabetes, history of previous MI, stroke, or coronary artery bypass grafting (CABG). Given the pharmacological differences between the ADP blockers in the speed of the onset of action in platelet inhibition, we also tested for possible treatment delay-associated interactions between ADP use and the outcome variables. A $p$ value $\leq 0.05$ was considered statistically significant. All analyses were performed using IBM SPSS Statistics version 25.0 (IBM Corporation, Armonk, NY, USA).

\section{Results}

During the study period, the mean age of patients arriving at the catheterization laboratory for primary PCI was 67.5 years. Of the 3218 total patients, 2277 (70.6\%) were male. The population characteristics among all patients and after stratification by ADP receptor blocker use are presented in Table 1 . The majority of patients $(95.2 \%, n=3070)$ were treated by PCI. Conservative treatment was determined optimal for $3.1 \%(n=101)$ of patients, and coronary bypass surgery was selected as the best option for $1.5 \%(n=47)$. The average mortality rate within 3 and 7 days from hospital admission was $4.2 \%$ and $5.4 \%$, respectively (Table 1 ).

The proportions of patients receiving the loading dose of ticagrelor, prasugrel, or clopidogrel were $47.6 \%$ $(n=1532), 22.1 \%(n=711)$, and $30.3 \%(n=975)$, respectively (Table 2). Clopidogrel was the most frequently used drug from 2007 to 2010, prasugrel from 2011 to 2014, and ticagrelor from 2014 to 2020 . There was an overlap between prasugrel and ticagrelor in 2014 and an overlap between prasugrel and clopidogrel between 2011 and 2013 (Fig. 1, Table 2). The time from treatment decision to first coronary expansion did not differ between the ADP receptor blockers. The proportion of patients treated with fibrinolytic therapy (and thus excluded from the study) decreased during the study period, from $58.2 \%$ in 2007 to only $0.9 \%$ in 2013 (Fig. 1, Table 2).

\subsection{Adenosine Diphosphate (ADP) Blocker Use and Culprit Artery Flow at Presentation and After Percutaneous Coronary Intervention}

The results of the adjusted binary logistic regression analysis suggest that loading the patient with ticagrelor or prasugrel rather than clopidogrel was associated with an increased likelihood of a moderate or good (grade 2-3) TIMI flow at presentation (observed in angiography before primary PCI; odds ratio [OR] 1.21, 95\% confidence interval [CI] 
Table 1 Study population characteristics between the ADP receptor blocker groups

\begin{tabular}{|c|c|c|c|c|c|}
\hline Variable & Total population & Clopidogrel & Prasugrel & Ticagrelor & $p$ value $^{\mathrm{a}}$ \\
\hline$N(\%)$ & 3218 & $975(30.3)$ & $711(22.1)$ & $1532(47.6)$ & \\
\hline Male & $2276(70.7)$ & $665(68.2)$ & $517(72.7)$ & $1094(71.4)$ & 0.096 \\
\hline Age, years [mean (SD)] & $67.50(12.79)$ & $68.91(13.16)$ & $65.91(12.22)$ & $67.35(12.71)$ & $<0.001$ \\
\hline Prevalence of diabetes & $563(17.5)$ & $181(18.6)$ & $92(12.9)$ & $290(18.9)$ & 0.001 \\
\hline Previous MI & $389(12.1)$ & $142(14.6)$ & $72(10.1)$ & $175(11.4)$ & 0.012 \\
\hline Previous stroke & $197(6.1)$ & $68(7.0)$ & $29(4.1)$ & $100(6.5)$ & 0.033 \\
\hline Previous PCI procedure & $318(9.9)$ & $97(9.9)$ & $71(10.0)$ & $150(9.8)$ & 0.986 \\
\hline Previous CABG procedure & $124(3.9)$ & $42(4.3)$ & $22(3.1)$ & $60(3.9)$ & 0.435 \\
\hline $\begin{array}{l}\text { Time }(\min ) \text { from treatment decision to first } \\
\text { coronary expansion [median (IQR)] }\end{array}$ & $76(60-100)$ & $75(59-103)$ & $78(63-101)$ & $76(60-99)$ & 0.293 \\
\hline \multicolumn{6}{|l|}{ TIMI pre-PCI ${ }^{\mathrm{b}}$} \\
\hline 0 & $1647(53.6)$ & $506(56.3)$ & $358(53.1)$ & $783(52.3)$ & 0.146 \\
\hline 1 & $245(8.0)$ & $74(8.2)$ & $59(8.8)$ & $112(7.5)$ & 0.563 \\
\hline 2 & $446(14.5)$ & $117(13.0)$ & $93(13.8)$ & $236(15.8)$ & 0.155 \\
\hline 3 & $732(23.8)$ & $201(22.4)$ & $164(24.3)$ & $367(24.5)$ & 0.473 \\
\hline Mean (SD) & 1.09 (1.28) & $1.01(1.26)$ & 1.09 (1.28) & $1.12(1.28)$ & 0.121 \\
\hline \multicolumn{6}{|l|}{ TIMI post-PCI ${ }^{\mathrm{b}}$} \\
\hline 0 & $117(3.8)$ & $37(4.1)$ & $26(3.9)$ & $54(3.6)$ & 0.814 \\
\hline 1 & $72(2.3)$ & $29(3.2)$ & $10(1.5)$ & $33(2.2)$ & 0.068 \\
\hline 2 & $226(7.4)$ & $74(8.2)$ & $62(9.2)$ & $90(6.0)$ & 0.015 \\
\hline 3 & $2655(86.5)$ & 758 (84.4) & $576(85.5)$ & $1321(88.2)$ & 0.022 \\
\hline Mean (SD) & $2.77(0.68)$ & $2.73(0.71)$ & $2.76(0.67)$ & $2.79(0.65)$ & 0.122 \\
\hline 3-day mortality & $136(4.2)$ & $58(5.9)$ & $25(3.5)$ & $53(3.5)$ & 0.006 \\
\hline 7-day mortality & $175(5.4)$ & $72(7.4)$ & $33(4.6)$ & $70(4.6)$ & 0.006 \\
\hline
\end{tabular}

Data are expressed as $n(\%)$ unless otherwise specified

$A D P$ adenosine diphosphate, $M I$ myocardial infarction, $P C I$ percutaneous coronary intervention, $C A B G$ coronary artery bypass grafting, $T I M I$ Thrombolysis in Myocardial Infarction, $S D$ standard deviation, IQR interquartile range

${ }^{a}$ Significance of differences between the ADP receptor blocker groups

${ }^{\mathrm{b}}$ Of patients included in the TIMI analysis (excluding patients treated conservatively or by coronary artery bypass grafting)

$1.03-1.42, p=0.022)$ (Fig. 2). In separate analyses, ticagrelor (but not prasugrel) use was associated with a significantly higher probability of a moderate or good flow compared with clopidogrel (Fig. 2); however, the comparison between ticagrelor and prasugrel did not reveal any statistically significant differences between these newer ADP blockers (OR $0.95,95 \%$ CI $0.79-1.15, p=0.591$ ).

The results for achieving moderate or good (grade 2-3) TIMI flow after PCI showed a similar tendency towards better results (significant differences in unadjusted analyses) (Table 1) in favor of the newer ADP blockers, but the differences were not statistically significant after adjusting for other factors associating with culprit artery flow (Fig. 2). As with the results for culprit artery flow before PCI, there was no difference between ticagrelor and prasugrel in culprit artery flow after PCI (OR 1.02, 95\% CI 0.68-1.53, $p=0.919)$.

Formal interaction testing did not reveal any significant interaction $(p>0.05)$ between treatment delay and ADP receptor blocker use on the TIMI flow of the culprit artery before or after PCI.

\subsection{ADP Blocker Use and Mortality}

With regard to acute mortality, use of the newer ADP blockers was associated with a combined lower mortality when compared with clopidogrel (OR 0.66 for 3-day mortality, 95\% CI 0.46-0.95; and OR 0.71 for 7-day mortality, $95 \%$ CI 0.52-0.98) (Fig. 3). In separate analyses, ticagrelor was associated with significantly lower mortality at both observation points when compared with clopidogrel (Fig. 3). The analyses for prasugrel alone showed a similar but non-significant trend (Fig. 3). There were no statistically significant differences between ticagrelor and prasugrel in 3-day mortality (OR $0.87,95 \%$ CI $0.51-1.37, p=0.481$ ) or 7-day mortality (OR $0.82,95 \%$ CI $0.53-1.27, p=0.384$ ).

The interaction analyses revealed a statistically significant interaction between treatment delay and use of the newer 
Table 2 Yearly number and percentage of patients treated with each of the three loading drugs, as well as patients receiving fibrinolytic therapy

\begin{tabular}{lllll}
\hline Year & Clopidogrel & Prasugrel & Ticagrelor & Fibrinolysis \\
\hline 2007 & $105(100)$ & 0 & 0 & $187(58.2)^{\mathrm{a}}$ \\
2008 & $172(100)$ & 0 & 0 & $141(39.8)^{\mathrm{a}}$ \\
2009 & $217(100)$ & 0 & 0 & $90(23.5)^{\mathrm{a}}$ \\
2010 & $206(90.4)$ & $22(9.6)$ & 0 & $49(13.2)^{\mathrm{a}}$ \\
2011 & $67(29.1)$ & $162(70.4)$ & $1(0.4)$ & $43(12.9)^{\mathrm{a}}$ \\
2012 & $71(27.1)$ & $191(72.9)$ & 0 & $36(10.5)^{\mathrm{a}}$ \\
2013 & $56(19.7)$ & $224(78.9)$ & $4(1.4)$ & $3(0.9)^{\mathrm{a}}$ \\
2014 & $34(12.5)$ & $108(39.7)$ & $130(47.8)$ & $2(0.6)^{\mathrm{a}}$ \\
2015 & $20(7.9)$ & $1(0.4)$ & $233(91.7)$ & 0 \\
2016 & $7(2.5)$ & 0 & $278(97.5)$ & $1(0.3)^{\mathrm{a}}$ \\
2017 & $8(2.5)$ & $1(0.3)$ & $317(97.2)$ & 0 \\
2018 & $8(3.4)$ & 0 & $227(96.6)$ & $1(0.3)^{\mathrm{a}}$ \\
2019 & $3(1.0)$ & $2(0.7)$ & $283(98.3)$ & 0 \\
2020 & $1(1.7)$ & 0 & $59(98.3)$ & 0 \\
Total & 975 & 711 & 1532 & 553 \\
\hline
\end{tabular}

Data are expressed as $n(\%)$

STEMI ST-elevation myocardial infarction

${ }^{\mathrm{a}} \mathrm{Of}$ all STEMI patients
ADP receptor blockers, associating with acute mortality ( $p<0.05$ for testing interaction for 3- and 7-day mortality). For this reason, the population was divided into two subgroups for further analysis: patients with a delay from the decision regarding reperfusion therapy to the first balloon inflation of more than $76 \mathrm{~min}$, and patients with a delay of less than this median. According to the regression analyses, in the subpopulation with shorter delays, the differences between the drugs were very distinct, with a more than $50 \%$ lower mortality risk associated with the use of the newer ADP blockers (ticagrelor and prasugrel combined). Among patients with longer delays, practically no difference was observed between the use of newer ADP blockers versus clopidogrel (Table 3 ).

\section{Discussion}

The observations of this retrospective register study suggest that the use of newer ADP receptor blockers (ticagrelor or prasugrel) in preprocedural loading is associated with lower acute mortality, as well as better preprocedural flow in the culprit artery, when compared with clopidogrel. After PCI, there were no significant differences in culprit artery flow

Fig. 1 Use of different preprocedural ADP receptor blockers in patients arriving to undergo a primary percutaneous coronary intervention for an STelevation myocardial infarction at Tays Heart Hospital during 2007-2020. Each bar represents patients over a 48-day period. Red represents clopidogrel, green represents prasugrel, and blue represents ticagrelor. $A D P$ adenosine diphosphate

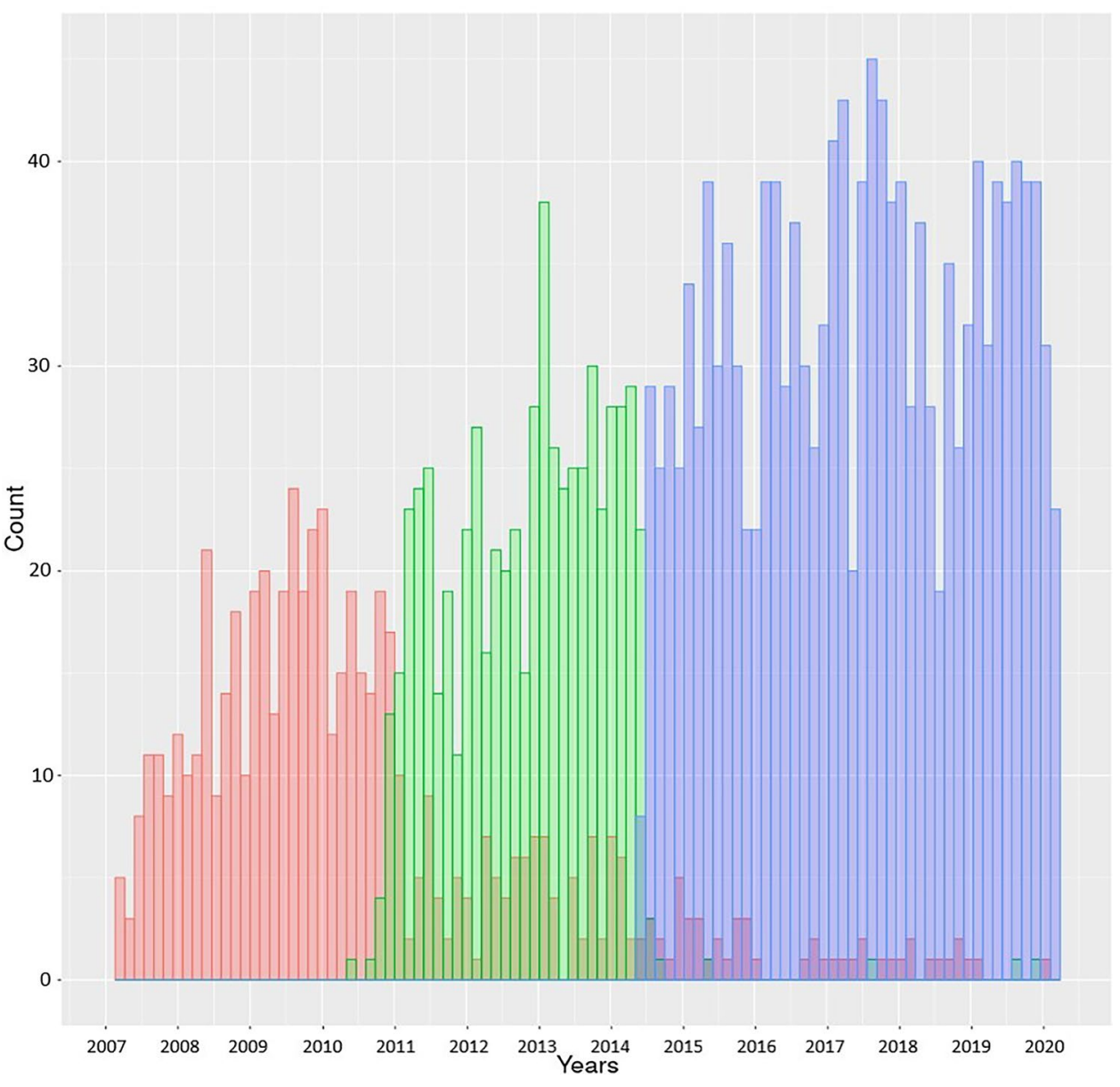


Fig. 2 The probability, associated with the preprocedural use of newer ADP receptor blockers, of achieving moderate or good culprit artery flow before and after percutaneous coronary intervention when compared with clopidogrel. $A D P$ adenosine diphosphate, TIMI Thrombolysis in Myocardial Infarction, $O R$ odds ratio, $C I$ confidence interval
TIMI pre: Ticagrelor: 1.23 (1.04-1.46)

Prasugrel: 1.18 (0.95-1.45)

Ticagrelor or Prasugrel: 1.21 (1.03-1.42)

TIMI post: Ticagrelor: $1.20(0.86-1.69)$

Prasugrel: 1.27 (0.83-1.95)

Ticagrelor or Prasugrel: 1.23 (0.90-1.68) 0.00 1.00

OR (95 \% C.I.) compared to Clopidogrel

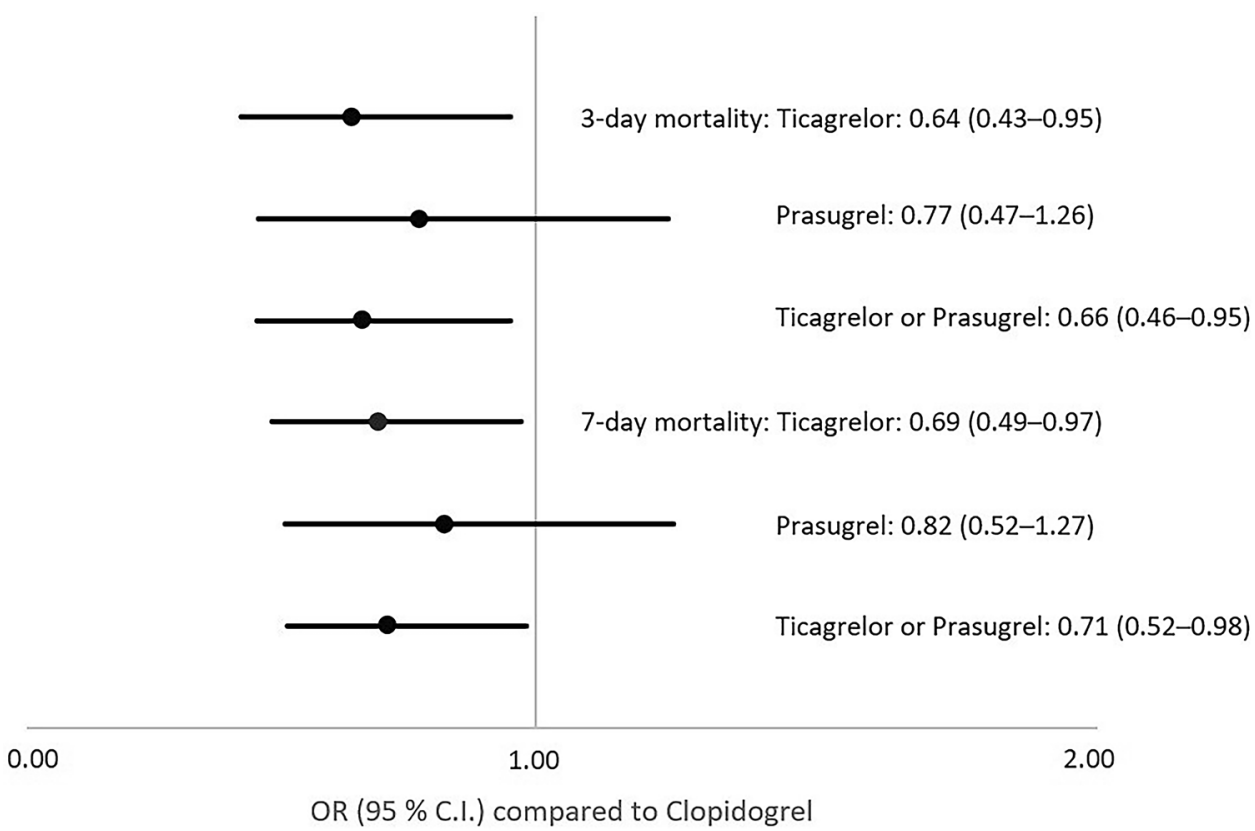

OR (95 \% C.I.) compared to Clopidogrel
Fig. 3 Odds ratios (95\% confidence interval) for 3 - and 7-day mortality for ticagrelor, prasugrel, and the combined population compared with clopidogrel. $\mathrm{OR}$ odds ratio, $\mathrm{CI}$ confidence interval between the ADP receptor blockers. We did not observe any significant differences between ticagrelor and prasugrel in any outcome. Our results also suggest that the differences in the therapeutic performance between the drugs is relative to the treatment delay, as we observed no difference between the use of the newer ADP blockers versus clopidogrel among patients with longer delays.

In a previously published propensity-matched analysis of a very large retrospective registry of over 89,000 patients in the UK, there were no significant differences in 30-day or 1-year mortality between ticagrelor and clopidogrel, but the use of prasugrel was associated with lower mortality when compared with the use of ticagrelor and clopidogrel in patients who underwent primary PCI for STEMI [18]. Furthermore, the results of the recent ISAR-REACT clinical trial comparing the use of ticagrelor and prasugrel in ACS also showed significantly better 1-year results for the use of prasugrel when compared with ticagrelor [14]; however, these results contradict the recent meta-analyses of more than 50,000 patients, as well as the recent PRAGUE study, in which there was no evidence of a significant difference between ticagrelor and prasugrel as regards either mortality 
Table 3 Results of the logistic regression analysis among the population, divided by the median delay from treatment decision to first coronary expansion [OR (95\% CI) for 3- and 7-day mortality compared with clopidogrel]

\begin{tabular}{|c|c|c|c|c|}
\hline \multirow[t]{3}{*}{ Variable } & \multicolumn{4}{|l|}{ Treatment delay } \\
\hline & \multicolumn{2}{|c|}{$\begin{array}{l}\text { Below the median of } \\
76 \text { min }\end{array}$} & \multicolumn{2}{|c|}{$\begin{array}{l}\text { Above the median of } \\
76 \text { min }\end{array}$} \\
\hline & OR $(95 \% \mathrm{CI})$ & $p$ value & OR $(95 \% \mathrm{CI})$ & $p$ value \\
\hline \multicolumn{5}{|c|}{ 3-day mortality } \\
\hline Ticagrelor & $0.37(0.19-0.72)$ & 0.004 & $0.95(0.48-1.88)$ & 0.875 \\
\hline Prasugrel & $0.43(0.17-1.10)$ & 0.077 & $0.84(0.33-2.13)$ & 0.708 \\
\hline $\begin{array}{l}\text { Ticargre- } \\
\text { lor and } \\
\text { prasugrel } \\
\text { com- } \\
\text { bined }\end{array}$ & $0.38(0.20-0.71)$ & 0.002 & $0.91(0.47-1.76)$ & 0.779 \\
\hline \multicolumn{5}{|c|}{ 7-day mortality } \\
\hline Ticagrelor & $0.49(0.27-0.90)$ & 0.021 & $1.03(0.56-1.89)$ & 0.933 \\
\hline Prasugrel & $0.45(0.19-1.09)$ & 0.076 & $1.23(0.56-2.70)$ & 0.601 \\
\hline $\begin{array}{l}\text { Ticargre- } \\
\text { lor and } \\
\text { prasugrel } \\
\text { com- } \\
\text { bined }\end{array}$ & $0.48(0.27-0.85)$ & 0.011 & $1.07(0.60-1.92)$ & 0.820 \\
\hline
\end{tabular}

$O R$ odds ratio, $C I$ confidence interval

or major cardiac adverse events [15-17]. Our results support the findings of the latter studies, as the use of the newer ADP blockers seems to be associated with lower acute mortality and better culprit artery flow at presentation when compared with clopidogrel, while there seems to be no significant difference between the newer ADP blockers in any of the tested outcomes.

Our observation of lower acute mortality among patients receiving newer ADP blockers is in line with the longer-term results of randomized controlled trials [9-11, 25, 26]. However, according to our results, this association is especially strong among patients with a relatively short treatment delay, but is reduced to insignificant among patients with longer delays. This suggests that the newer ADP receptor blockers, which have a relatively rapid onset of antiplatelet action [27-29], are associated with lower mortality when invasive treatment is available at an early stage. Supporting this, the efficacy of primary PCI treatment in STEMI is better when treatment delays are shorter [30]. Although we cannot rule out the possibility of type I error regarding this finding, it is plausible that when treatment delays grow longer, the relative efficacy of the newer ADP blockers also decreases along with the overall efficacy of the treatment.

Despite the clear association between ADP receptor blocker use and acute mortality, we did not observe significant differences between any of the drugs in procedural success, defined by TIMI 2-3 culprit artery flow after PCI.
A clear tendency towards better results in favor of the newer ADP blockers was observed, but after adjusting for potential confounding factors, the association became non-significant. However, this analysis was only conducted among patients with attempted PCI, and it is plausible that the early mortality associated with the use of clopidogrel can cause selection bias in the analyses. Corresponding with the findings of lower early mortality, we also observed that patients treated with the newer ADP receptor blockers had better culprit artery flow at presentation.

Interestingly, we did not observe any significant difference between the use of ticagrelor and prasugrel regarding any of the endpoints. While ticagrelor was associated with significantly lower mortality when compared with clopidogrel, the analyses between prasugrel and clopidogrel showed a similar, but statistically non-significant, association in favor of prasugrel. The reason for the statistically non-significant finding of a similar association is most likely a sample size issue in the analyses, caused by the lower number of patients treated with prasugrel $(n=711)$ than with ticagrelor $(n=1532)$. Overall, our observations suggest that both of these ADP receptor blockers can probably be used in acute care with similar results. Furthermore, given the fact that ticagrelor is not contraindicated in patients with a prior stroke or transient ischemic attack [3], our results support the use of ticagrelor as an acute-phase loading drug in patients with STEMI, as there is no direct indication of prasugrel outperforming ticagrelor in regard to acute mortality.

There are multiple confounding factors in our study that could not be considered in our analysis. First of all, the frequency of patients treated with fibrinolytic therapy decreased drastically during the study. The majority of patients who underwent fibrinolytic therapy were treated during the period when clopidogrel was the go-to ADP receptor blocker, which could cause selection bias. However, the reason for choosing fibrinolytic therapy was, in most instances, not medical but rather logistic, as the STEMI network was introduced gradually in different parts of the hospital district. Similarly, as this study was not a randomized controlled trial, we cannot exclude the possibility of confounding by selection bias during the periods when the use of different ADP receptor blockers overlapped. However, the choice of loading drug, even during those times, was largely based on the availability of the drugs in ambulances in different areas of the STEMI network during the transition. For this reason, no formal propensity matching was performed (electronic supplementary Figs. 1 and 2)

Additionally, as the present results are based on singlecenter experience, the size of the population in our study is limited. It is possible that, with a larger sample size, some trends found in the study could have proven to be statistically significant, such as when comparing the probability of a successful procedure between the groups. However, in a 
single center, it is easier to compare results for one variable, as other factors can be more easily controlled for and there are no center-related differences in cardiac care.

Finally, as we did not have full data on the patients' discharge medication or follow-up data on the use of prescribed medication after discharge, we were unable to provide reliable estimates of the association between ADP blocker use and long-term mortality among STEMI patients. However, in exploratory analyses, the association between lower longterm mortality and the use of newer ADP blockers was similar to the results regarding acute mortality (electronic supplementary Table 1). Due to the lack of follow-up data on the use of these medications, we elected to primarily report the results for acute mortality. The short-term treatment result associated with the use of different ADP blockers is the most reliable endpoint because little else affecting acute mortality has changed in acute care between 2007 and 2020, while patients in this study population were treated. For example, although the use of bare metal stents has been mostly replaced by the use of drug-eluting stents, this change has had a minimal to no effect on short-term mortality.

\section{Conclusions}

In STEMI patients with short treatment delays, prehospital loading with ticagrelor or prasugrel is associated with lower acute mortality when compared with clopidogrel. Treating a patient with one of the newer ADP receptor blockers also associates with an increased probability of moderate or good blood flow in the culprit artery even before the PCI procedure. There is no significant difference between ticagrelor and prasugrel in any of the outcomes.

Supplementary Information The online version contains supplementary material available at https://doi.org/10.1007/s40261-021-01045-2.

\section{Declarations}

Funding This study was supported by Competitive State Research Financing of the Expert Responsibility Area of Tampere University Hospital, in addition to the Tampere University Hospital support association and Business Finland research funding (Grant 4197/31/2015) as part of a collaboration between Tays Heart Hospital, Tampere University, VTT Technical Research Centre of Finland Ltd, Politecnico di Milano, GE Healthcare Finland Ltd, Fimlab Laboratories Ltd, and Bittium Medanalytics Ltd. MH has been supported by the Finnish Foundation for Cardiovascular Research (Grant 200076). TL has been supported by the Academy of Finland (Grants 286284 and 322098), the Finnish Society of Clinical Chemistry, Competitive State Research Financing of the Expert Responsibility Area of Tampere University Hospital (Grant X51001), Finnish Foundation for Cardiovascular Research, Tampere Tuberculosis Foundation, Emil Aaltonen Foundation, Yrjö Jahnsson Foundation, Signe and Ane Gyllenberg Foundation, Diabetes Research Foundation of the Finnish Diabetes Association, Tampere University Hospital Support Foundation, and the EU Horizon 2020 (Grants 755320 and 848146).
Conflict of interest Markus Hautamäki, Leo-Pekka Lyytikäinen, Markku Eskola, Terho Lehtimäki, Kjell Nikus, Niku Oksala, Juho Tynkkynen, and Jussi Hernesniemi declare they have no conflicts of interest.

Ethical approval As this was a retrospective registry study, formal ethical approval was not required. However, the study was conducted according to the ethical principles of the Declaration of Helsinki on the use of human data and approved by the Institutional Review Board overseeing the use of registry data, as required by Finnish legislation.

Consent to participate Not applicable.

Consent for publication Not applicable.

Availability of data and material The anonymized data from this study are available upon request and for justified reasons.

Code availability Not applicable.

Author contributions All authors significantly and equally contributed to the planning, conducting, and reporting of this study. $\mathrm{MH}$ and $\mathrm{JH}$ are the authors responsible for the overall content of this study.

Open Access This article is licensed under a Creative Commons Attribution-NonCommercial 4.0 International License, which permits any non-commercial use, sharing, adaptation, distribution and reproduction in any medium or format, as long as you give appropriate credit to the original author(s) and the source, provide a link to the Creative Commons licence, and indicate if changes were made. The images or other third party material in this article are included in the article's Creative Commons licence, unless indicated otherwise in a credit line to the material. If material is not included in the article's Creative Commons licence and your intended use is not permitted by statutory regulation or exceeds the permitted use, you will need to obtain permission directly from the copyright holder. To view a copy of this licence, visit http://creativecommons.org/licenses/by-nc/4.0/.

\section{REFERENCES}

1. Gurbel PA, Bliden KP, Hiatt BL, O'Connor CM. Clopidogrel for coronary stenting. Circulation. 2003;107(23):2908-13.

2. Brilakis ES, Patel VG, Banerjee S. Medical management after coronary stent implantation: a review. JAMA. 2013;310(2):189-98.

3. Valgimigli M, Bueno H, Byrne RA, et al. 2017 ESC focused update on dual antiplatelet therapy in coronary artery disease developed in collaboration with EACTS: the Task Force for dual antiplatelet therapy in coronary artery disease of the European Society of Cardiology (ESC) and of the European Association for Cardio-Thoracic Surgery (EACTS). Eur Heart J. 2018;39(3):213-60.

4. Gregorini L, Marco J, Fajadet J, et al. Ticlopidine and aspirin pretreatment reduces coagulation and platelet activation during coronary dilation procedures. J Am Coll Cardiol. 1997;29(1):13-20.

5. Sibbing D, Kastrati A, Berger PB. Pre-treatment with P2Y12 inhibitors in ACS patients: who, when, why, and which agent? Eur Heart J. 2016;37(16):1284-95.

6. Bonello L, Camoin-Jau L, Arques S, et al. Adjusted clopidogrel loading doses according to vasodilator-stimulated phosphoprotein phosphorylation index decrease rate of major adverse cardiovascular events in patients with clopidogrel resistance. J Am Coll Cardiol. 2008;51(14):1404. 
7. Parodi G, Bellandi B, Valenti R, Migliorini A, Marcucci R, Carrabba N, Giurlani L, Gensini GF, Abbate R, Antoniucci D. Comparison of double $(360 \mathrm{mg})$ ticagrelor loading dose with standard $(60 \mathrm{mg})$ prasugrel loading dose in ST-elevation myocardial infarction patients: the Rapid Activity of Platelet Inhibitor Drugs (RAPID) primary PCI 2 study. Am Heart J. 2014;167(6):909-14.

8. Ibanez B, James S, Agewall S, et al. 2017 ESC Guidelines for the management of acute myocardial infarction in patients presenting with ST-segment elevationThe Task Force for the management of acute myocardial infarction in patients presenting with STsegment elevation of the European Society of Cardiology (ESC). Eur Heart J. 2018;39(2):119-77.

9. Wallentin L, Becker RC, Budaj A, et al. Ticagrelor versus clopidogrel in patients with acute coronary syndromes. N Engl J Med. 2009;361(11):1045-57.

10. Cannon CP, Harrington RA, James S, Ardissino D, Becker RC, Emanuelsson H, Husted S, Katus H, Keltai M, Khurmi NS, Kontny F, Lewis BS, Steg PG, Storey RF, Wojdyla D, Wallentin L. Comparison of ticagrelor with clopidogrel in patients with a planned invasive strategy for acute coronary syndromes (PLATO): a randomised double-blind study. Lancet. 2010;375(9711):283-93.

11. Wiviott SD, Braunwald E, McCabe $\mathrm{CH}$, et al. Prasugrel versus clopidogrel in patients with acute coronary syndromes. N Engl J Med. 2007;357(20):2001-15.

12. Steg PG, Stefan J, Harrington RA, et al. Ticagrelor versus clopidogrel in patients with ST-elevation acute coronary syndromes intended for reperfusion with primary percutaneous coronary intervention. Circulation. 2010;122(21):2131-41.

13. Montalescot G, Wiviott SD, Braunwald E, et al. Prasugrel compared with clopidogrel in patients undergoing percutaneous coronary intervention for ST-elevation myocardial infarction (TRITON-TIMI 38): double-blind, randomised controlled trial. Lancet. 2009;373(9665):723-31.

14. Schüpke S, Neumann F, Menichelli M, et al. Ticagrelor or prasugrel in patients with acute coronary syndromes. N Engl J Med. 2019;381(16):1524-34.

15. Zuzana M, Ota H, Roman M, et al. Prasugrel versus ticagrelor in patients with acute myocardial infarction treated with primary percutaneous coronary intervention. Circulation. 2016;134(21):1603-12.

16. Wang Z, Zhou D, Su Y, Si L, Xu Q. Prasugrel or ticagrelor relative to clopidogrel in triple-antiplatelet treatment combined with glycoprotein IIb/IIIa inhibitor for patients with STEMI undergoing PCI: a meta-analysis. BMC Cardiovasc Disord. 2020;20(1):130.

17. Khan MS, Memon MM, Usman MS, et al. Prasugrel vs. ticagrelor for acute coronary syndrome patients undergoing percutaneous coronary intervention: a systematic review and meta-analysis. Am J Cardiovasc Drugs. 2019;19(5):465-76.
18. Olier I, Sirker A, Hildick-Smith DJR, et al. Association of different antiplatelet therapies with mortality after primary percutaneous coronary intervention. Heart. 2018;104(20):1683-90.

19. Hernesniemi JA, Mahdiani S, Lyytikäinen LP, Lehtimäki T, Eskola M, Nikus K, et al. Cohort description for MADDECmass data in detection and prevention of serious adverse events in cardiovascular disease. In: Eskola H, Väisänen O, Viik J, Hyttinen J (eds) EMBEC and NBC 2017. IFMBE proceedings, Vol 65. Springer, Singapore.

20. Syyli N, Hautamäki M, Antila K, et al. Left ventricular ejection fraction adds value over the GRACE score in prediction of 6-month mortality after ACS: the MADDEC study. Open Heart. 2019;6(1):e001007.

21. Hautamäki M, Lyytikäinen L, Mahdiani S, et al. The association between charlson comorbidity index and mortality in acute coronary syndrome - the MADDEC study. Scand Cardiovasc J. 2020;54(3):146-52.

22. Hernesniemi JA, Mahdiani S, Tynkkynen JA, et al. Extensive phenotype data and machine learning in prediction of mortality in acute coronary syndrome-the MADDEC study. Ann Med. 2019;51(2):156-63.

23. Salomaa V, Pääkkönen R, Pietilä A. Suomalaisen Kuoleman Trendit. Suom Laakaril. 2009;9:780-1.

24. Tilastokeskus. Laatuseloste: Kuolemansyyt 2016. https://www. stat.fi/til/ksyyt/2016/ksyyt_2016_2017-12-29_laa_001_fi.html\#1. Tilastotietojen relevanssi. Accessed 12 Oct 2018.

25. James S, Angiolillo DJ, Cornel JH, et al. Ticagrelor vs clopidogrel in patients with acute coronary syndromes and diabetes: a substudy from the PLATelet inhibition and patient Outcomes (PLATO) trial. Eur Heart J. 2010;31(24):3006-16.

26. Steg PG, James S, Harrington RA, et al. Ticagrelor versus clopidogrel in patients with ST-elevation acute coronary syndromes intended for reperfusion with primary percutaneous coronary intervention: A Platelet Inhibition and Patient Outcomes (PLATO) trial subgroup analysis. Circulation. 2010;122(21):2131-41.

27. Raju NC, Eikelboom JW, Hirsh J. Platelet ADP-receptor antagonists for cardiovascular disease: past, present and future. Nat Clin Pract Cardiovasc Med. 2008;5(12):766-80.

28. Teng R. Ticagrelor: pharmacokinetic, pharmacodynamic and pharmacogenetic profile: an update. Clin Pharmacokinet. 2015;54(11):1125-38.

29. Roffman DS. Developments in oral antiplatelet agents for the treatment of acute coronary syndromes: clopidogrel, prasugrel, and ticagrelor. J Pharm Pract. 2016;29(3):239-49.

30. Żurowska-Wolak M, Piekos P, Jąkała J, Mikos M. The effects of prehospital system delays on the treatment efficacy of STEMI patients. Scand J Trauma Resusc Emerg Med. 2019;27(1):39. 